\title{
Impacto das tecnologias educativas no controle da asma: uma revisão sistemática da literatura
}

\section{Impact of educational technology in asthma control: a systematic review of the literature}

\author{
Andrea Edwiges Pinheiro de Menezes Barreto¹. Francisco Ranilson Alves Silva². \\ 1 Residência em Pediatria, Hospital Universitário Walter Cantídio, Universidade Federal do Ceará (UFC), Fortaleza, Ceará, Brasil. \\ 2 Pediatra, Hospital Universitário Walter Cantídio, Fortaleza, Ceará, Brasil.
}

\section{RESUMO}

O estudo apresenta uma revisão de literatura sobre o controle da asma em crianças, demonstrando as principais tecnologias educativas utilizadas no controle dessa patologia. O objetivo geral do estudo consiste em conhecer quais as tecnologias educativas e qual impacto essas tecnologias causam no tratamento e controle da asma. Dentre os objetivos específicos do estudo destaca-se a possibilidade de garantir informações necessárias aos profissionais de saúde e pacientes, tais como, o reconhecimento de sinais, sintomas e fatores agravantes e como evitá-los. Foi utilizado revisão de literatura nacional e internacional, selecionando artigos publicados nos últimos dez anos, incluindo diretrizes, periódicos e editoriais. O estudo garante a importância da padronização de aplicação educacional que leve a racionalização dos itens abordados, facilitando o reconhecimento das dificuldades dos pacientes, além de melhora na transferência de conhecimento pelo profissional de saúde ao paciente, e conscientização das intervenções necessárias. Dessa maneira, garante orientações de ações possíveis de realizar com controle da asma, e consequentemente, com melhora significativa na qualidade de vida, diminuição da morbidade e diminuição da mortalidade das crianças com essa patologia.

Palavras-chave: Asma. Tecnologias educativas. Dispnéia.

\section{ABSTRACT}

The study presents a literature review on the control of asthma in children, showing the main educational technologies used in the control of this disease. The overall objective of the study is to understand what educational technologies and what impact these technologies have on the treatment and management of asthma. The specific goals of the study there is the possibility to ensure necessary information to health professionals and patients, such as the recognition of signs, symptoms and aggravating factors and how to avoid them. We used national and international literature review, selecting articles published in the last ten years, including guidelines, journals and editorial. The study ensures the importance of standardization of educational application that takes the rationalization of covered items, facilitating recognition of the difficulties of patients and improvement in the professional knowledge transfer to patient health, and awareness of the necessary interventions. In this way ensures guidelines of possible actions to perform with asthma control, and consequently, with significant improvement in quality of life, decreased morbidity and decreased mortality of children with this disease.

Keywords: Asthma. Educational technology. Dyspnea.

Autor correspondente: Andrea Edwiges Pinheiro de Menezes Barreto, Rua Rotary, 330, Fortaleza, Ceará. CEP: 60455490 . Telefone: +5585 988166023. E-mail: andreapmb@hotmail.com

Conflito de interesses: Não há qualquer conflito de interesses por parte de qualquer um dos autores.

Recebido em: 24 Abr 2015; Revisado em: 02 Jul 2015; Aceito em: 13 Jul 2015. 


\section{INTRODUÇÃO}

A asma é uma doença inflamatória crônica que se caracteriza por sinais e sintomas recorrentes relacionados à hiperresponsividade das vias aéreas (HVA). ${ }^{1}$

As manifestações clínicas da asma podem incluir sibilos, dispnéia, opressão torácica e tosse, principalmente à noite ou no início da manhã. ${ }^{2}$

Os episódios de exacerbação da doença podem ser desencadeados por vários fatores, dentre eles estão infecções virais e bacterianas, fungos, poeiras, mudanças climáticas, umidade e aspectos emocionais. ${ }^{3}$

A asma é uma das condições crônicas mais comuns que acomete tanto crianças como adultos, sendo considerado um problema mundial de saúde. A prevalência de asma entre escolares brasileiros atinge valores de até $(25 \%)$, sendo a terceira causa $(2,6 \%)$ de internações hospitalares entre crianças e adolescentes no Brasil, cifras cearenses permanecendo em torno de $22,6 \%$ de asma ativa em escolares do município de Fortaleza. $^{4}$

As causas da asma englobam uma combinação de fatores como exposições ambientais, vulnerabilidades biológicas e genéticas inerentes. Os determinantes da história natural da asma ainda não são totalmente estabelecidos, não sendo possível definir claramente o prognóstico. Existem vários mecanismos imunopatológicos subjacentes que lactentes e crianças pré-escolares podem ser vinculados, levando a diminuição e limitação ao fluxo aéreo. Assim, ainda não é possível predizer com segurança a evolução clínica da sibilância entre lactentes e pré-escolares. ${ }^{5}$

O processo fisiopatológico da asma está associado a fatores genéticos e ambientais. Esses fatores desencadeiam a liberação de mediadores inflamatórios, resultantes da interação entre os linfócitos T CD4 tipo Th2 e os alérgenos. ${ }^{3}$ As células inflamatórias, mediadores inflamatórios e componentes estruturais celulares, implicados na patogenia da asma, promovem aumento da permeabilidade vascular, alteração na função mucociliar, hipersecreção de muco e constricção da musculatura lisa peribronquiolar. Os principais componentes que orquestram tais modificações são mastócitos, macrófagos, linfócitos, eosinófilos, neutrófilos e células epiteliais. A inflamação crônica e repetida poderá promover alterações permanentes das vias aéreas através de um processo de remodelamento, levando um quadro de irreversibilidade da obstrução, como é visto em alguns pacientes. ${ }^{6}$

A asma frequentemente é uma doença de diagnóstico difícil por ter inicio algumas vezes durante o primeiro ano de vida, sendo a sibilância respiratória um sintoma bastante comum de outras doenças prevalentes nessa faixa etária. Dessa maneira, a asma é considerada um diagnóstico de exclusão para crianças dessa idade. ${ }^{1}$

O diagnóstico clínico da asma em sua forma clássica de apresentação não é considerado tão difícil, mas algumas vezes é necessário confirmação por um método objetivo, uma vez que os sinais e sintomas da asma não são exclusivos dessa doença. Os testes diagnósticos disponíveis na prática clínica englobam espirometria, testes de broncoprovocação e medidas seriadas de prova de função pulmonar (PFE). Observa-se que em alguns casos, a comprovação da reversibilidade da obstrução ao fluxo aéreo pode ser evidenciada apenas com o teste terapêutico com corticóide oral. ${ }^{2}$

Em crianças até os cinco anos de idade o diagnóstico deve ser baseado em aspectos clínicos diante das dificuldades de conseguir medidas objetivas. Desse modo, a investigação deve ser baseada em critérios clínicos, antecedentes pessoais, história familiar e evolução dos sintomas e achados físicos. Entre as manifestações mais sugestivas de asma nessa faixa etária estão: episódios frequentes de sibilância; tosse ou sibilos que ocorrem à noite ou cedo pela manhã, desencadeados por riso ou choro intensos ou exercício físico; tosse sem relação evidente com viroses respiratórias; presença de atopia, especialmente rinite alérgica ou dermatite atópica; história familiar de asma e atopia; boa resposta clínica a $\beta 2$ agonistas inalatórios, associados ou não a corticóides orais ou inalatórios. $^{2}$

Atualmente, a asma pode ser classificada de acordo com o nível de controle da doença com objetivo de reduzir riscos futuros e evitar limitações clínicas. Essa classificação compreende analisar os seguintes parâmetros: sintomas diurnos, limitação de atividades, sintomas e despertares noturnos, necessidade de medicação de alívio e níveis de função pulmonar. Dessa maneira, a asma pode ser classificada em asma controlada, asma parcialmente controlada e asma não controlada. Os instrumentos usados de classificação compreendem em questionários adaptados à língua portuguesa. ${ }^{1}$

A doença pode ser controlada na maioria dos pacientes, diminuindo a apresentação dos sintomas diurnos e noturnos, o número de hospitalizações e melhora da função pulmonar. $\mathrm{O}$ controle da doença pode ser feito através de medidas educativas e com uso de medicamentos. Uma vez não controlada, a asma pode tornar-se crônica com limitação permanente ao fluxo aéreo, levando à limitação significativa física e social e até causar morte por crises graves. ${ }^{1}$ Os avanços no controle da asma, especialmente na farmacoterapia e nas tecnologias educativas, possibilitam que todos vivam normalmente, ainda que a criança tenha uma asma grave. ${ }^{5}$

É de suma importância que a asma seja conduzida de maneira eficiente a fim de minimizar as repercussões que essa doença pode gerar na vida dos pacientes e dos seus familiares. A educação juntamente com o tratamento farmacológico compreende o fundamento do manejo da asma. Pode-se observar que as intervenções educacionais associada a um plano de automanejo, possibilitam melhor controle da asma, reduz hospitalizações e diminui as faltas escolares. O manejo educativo compreende o entendimento do processo saúdedoença da asma e do correto uso dos dispositivos para que se obtenha um controle, evitando as crises muito graves e 
o empoderamento do cuidar. Há tendências atuais de fazer essa educação, com tecnologias e inovações em saúde, compreendendo desdes cartinhas, jogos à softwares. ${ }^{2}$

O tratamento da asma pode ser estabelecido de acordo com o nível de controle da asma, sendo dividido em cinco etapas de acordo com o tratamento atual e o seu nível de controle. Entre as medidas farmacológicas, estão os corticóides inalatórios (dose baixa, moderada e alta), $\beta 2$ - agonista de ação prolongada, corticóide oral, teofilina de liberação lenta e tratamento com anti-IgE. Esses medicamentos são direcionados de acordo com os níveis de controle e são agrupados ao longo das cinco etapas do tratamento. ${ }^{1}$

\section{PERGUNTA}

Qual impacto das tecnologias educativas no controle da asma?

\section{OBJETIVOS}

\section{- Objetivos Gerais}

Conhecer estudos sobre instrumentos educativos no controle da asma

\section{- Objetivos Específicos}

a) Fornecer aos profissionais de saúde informações atuais sobre os principais instrumentos educativos a fim de ajudar o controle da asma e diminuir exacerbações e hospitalizações devido à doença.

b) Possibilitar aos pacientes e familiares o reconhecimento dos sintomas, reconhecimento dos fatores desencadeantes e de como evitá-los.

\section{MÉTODO}

Foi realizada revisão da literatura nacional e internacional utilizando os bancos de dados MEDLINE (PUBMED), LILACS-BIREME, sendo selecionados artigos publicados nos últimos dez anos, abordando a doença asma com ênfase em controles e métodos tecnológicos educativos utilizados no controle dessa patologia. Os seguintes termos de pesquisa (palavras-chaves e delimitadores) foram utilizados em várias combinações: 1) asma; 2) controle; 3) tecnologias educativas.

A pesquisa bibliográfica incluiu artigos originais, artigos de revisão, editoriais e diretrizes escritos nas línguas inglesa, espanhola e portuguesa, sendo selecionados de acordo com os critérios do Centro Oxford de Evidência (2011).

O início da procura das bases de dados aconteceu a partir do Portal das Capes, preenchendo as caixas da página da base de dados com os descritores supracitados, usando os boleanos OR e AND.

Foram aceitos (critérios de inclusão) para essa revisão os trabalhos que analisaram intervenções educativas em asma na infância; relataram avaliação quantitativa dos resultados das intervenções (antes e depois da intervenção); utilizaram um ou mais dos seguintes parâmetros de avaliação: nível de conhecimento da doença, capacidade de automanejo, função pulmonar e indicadores de mortalidade. Entre os indicadores de mortalidade, foram avaliados os seguintes aspectos: sintomas diurnos e noturnos, absenteísmo escolar, índice de qualidade de vida, status funcional (grau de restrição nas atividades físicas habituais) e frequência de utilização de serviços de saúde.

Os critérios de exclusão foram: ausência de resultados quantificados após as intervenções; parâmetros de avaliação diferentes dos referidos no tópico "critérios de inclusão"; alvo em apenas adultos asmáticos, isto é, não incluindo crianças no estudo; alvo em, simultaneamente, crianças asmáticas e crianças com outras patologias pulmonares ou outras doenças crônicas associadas.

\section{DISCUSSÃO}

A educação deve ajudar o paciente, seus familiares e cuidadores na aquisição de motivações, habilidades e confiança, facilitar o reconhecimento dos sintomas, o conhecimento dos fatores desencadeantes e de como evitá-los, além de tornar a participação do paciente ativa no tratamento. Deve ser informado à população em geral que a asma é uma doença pulmonar crônica e, se adequadamente tratada, pode ser controlada, permitindo uma vida normal. Informar escolas, colônias de férias, seguradores de saúde e empresas públicas e privadas, para que possam identificar a asma e encaminhar o paciente a tratamento. $O$ papel da educação deve garantir a informação dos profissionais da saúde para estabelecer o diagnóstico e a abordagem terapêutica adequada. ${ }^{1}$

A educação é eficaz e recomendada como parte fundamental do plano de tratamento da asma. Ela reduz hospitalizações, diminui o absenteísmo escolar, reduz número de visitas à emergência, diminui os sintomas noturnos, melhora a qualidade de vida e, consequentemente, diminui a morbidade da doença. A educação fornecida ao paciente melhora a utilização da técnica inalatória, principalmente, quanto ao uso dos inaladores dosimetrados. ${ }^{7}$

A educação na asma para profissionais de saúde e para pacientes melhora o reconhecimento dos sintomas, ajuda a evitar exacerbações, garante melhor tratamento e melhor qualidade de vida. ${ }^{8}$ Dessa forma, o tratamento farmacológico não é a única maneira de controlar a asma. Apesar da asma não ter cura, a gestão adequada do tratamento, dando ênfase às medidas educacionais, pode controlar a doença e permitir que as crianças desfrutem de boa qualidade de vida. ${ }^{9}$

A aderência é utilizada para descrever a concordância entre a prescrição da terapia, incluindo medidas educacionais e medicação, e a aplicação desta prescrição pelo paciente no seu processo terapêutico..$^{10}$

Pode-se observar que a aderência possui três componentes: a aceitação das recomendações, a observação da prescrição pelo uso da terapia e persistência na aplicação das recomendações. ${ }^{11}$ Algumas causas podem ser discriminadas para não aderência 
ao tratamento, tais como, a ansiedade em relação aos efeitos colaterais, a negação da doença, desconforto com o uso da terapia inalatória, treino inadequado, dificuldade em compreender a necessidade do uso prolongado, custo da medicação, dificuldade em obter regularmente a medicação, fatores educacionais e culturais. ${ }^{12}$

A perda da aderência do paciente resulta na perda do controle da asma, no aumento da mortalidade e mortalidade da doença, assim como, no aumento das visitas à emergência. As causas da não aderência podem ser divididas em causa não intencional, por esquecimento ou má compreensão das prescrições, ou causa intencional, quando o paciente escolhe não seguir a prescrição. ${ }^{12}$

É bastante conhecida a dificuldade à terapia com corticóide inalatório (CI), oscilando a aderência em crianças de $48 \%$ a $55 \%$ e sendo influenciada pela percepção da gravidade da doença. ${ }^{12}$ Alguns estudos mostraram que a combinação do CI com beta agonista de longa ação (LABA) possui melhor aderência do que apenas o uso isolado do primeiro. ${ }^{13}$

Em 2005, Santana et al, realizaram uma revisão analítica da literatura visando avaliar as internações educativas para asmáticos de 0 a 18 anos, no período de 1992 a 2002, e identificar características relacionadas com sua eficácia. Os parâmetros de avaliação usados pelos estudos foram: variáveis de morbidade, uso de serviços de saúde, qualidade de vida, função pulmonar, conhecimento sobre a doença e habilidades de automanejo. Em 32 estudos (82\%), relatou-se benefício sobre uma ou mais variáveis. Entre os 27 estudos controlados, $85,7 \%$ produziram melhora nas habilidades de automanejo, $83,3 \%$ no conhecimento, $80 \%$ nos sintomas diurnos ou noturnos, 71,4\% nas visitas médicas não programadas, $66,6 \%$ na capacidade para atividades físicas, 54,5\% nas hospitalizações, $50 \%$ nas visitas à emergência, $50 \%$ na função pulmonar, 22,2\% no absenteísmo escolar e $20 \%$ na qualidade de vida. Esse estudo concluiu que o número de itens de conteúdo foi a única característica dos programas educativos revisados associada ao nível de eficácia. Técnicas educativas sofisticadas não contribuíram para melhores resultados. A educação de crianças e adolescentes asmáticos produz resultados benéficos, mas são necessários estudos com melhor controle de variáveis confundidoras, para uma avaliação mais precisa da sua eficácia. ${ }^{14}$

Em 2006, Chahtin et al, avaliaram a adesão ao tratamento preventivo de asma persistente moderada e grave. Nesse estudo, foram incluídos 135 pacientes contatados por telefone por um profissional treinado, sendo o primeiro telefonema para registro de dados e o último em 90 dias para avaliar a adesão ao tratamento. Ao final, foi observada uma taxa de adesão de 51,9\%. Entre os asmáticos persistentes moderados foi de $43 \%$ e entre os persistentes graves de $64 \%$, observandose que quanto mais grave a asma, maior foi a taxa de adesão observada. $^{15}$

Em 2007, Araujo et al em um estudo com 77 pacientes com asma grave, dos quais, $40 \%$ tinham asma de difícil controle, encontrou que em $68 \%$ dos casos de difícil controle, o principal fator contribuinte foi a não adesão ao tratamento. ${ }^{16}$

Em 2007, Costa et al, realizaram um estudo observacional com o objetivo de avaliar o impacto de um programa de acampamento educacional para crianças asmáticas, com duração de cinco dias, em termos de melhora do nível de conhecimento sobre a asma e da melhora da destreza no uso de medicação inalatória e na execução de exercícios físicos. Diariamente, as crianças recebiam 20 minutos de educação interativa, a técnica do uso do inalador dosimetrado era revista, realizavam-se duas medidas de pico de fluxo, e as crianças realizavam atividades físicas que incluíam exercícios respiratórios e de relaxamento. Um questionário que avaliava o conhecimento das crianças sobre a asma, os desencadeadores da crise, a medicação, os conceitos errôneos e o uso de espaçadores foi aplicado antes e após a intervenção. $\mathrm{O}$ uso correto da medicação inalatória e os sintomas relacionados às atividades físicas também foram avaliados antes e após a intervenção. Esse estudo mostrou que $25 \%$ das crianças avaliadas apresentaram melhora do nível de conhecimento especifico sobre a asma e melhora dos escores de dispnéia relacionada a atividade física. Dessa maneira, o programa de acampamento educacional pode aumentar o conhecimento sobre questões específicas, encorajar a participação em atividades físicas e melhorar a habilidade das crianças no manejo da asma. ${ }^{17}$

Em 2008, Angelini et al, avaliaram o conhecimento da doença e a melhora clinica de portadores de asma persistente moderada e grave, aplicando um programa de educação para 164 pacientes. Os pacientes foram acompanhados por dois anos em ambulatório. O programa de educação ofereceu aulas de fisiopatologia, controle ambiental, medicamentos e treinamento da técnica inalatória. A intervenção educacional aumentou o conhecimento da doença significativamente, trouxe melhora clínica, diminuição do uso de corticóide oral, redução de visitas à emergência e menor número de faltas à escola. $^{18}$

Em 2008, Silveira et al verificaram a adequação da assistência médica prestada a pacientes asmáticos do Sistema Único de Saúde (SUS) de acordo com diretrizes internacionais para o manejo da asma. Nesse estudo, foram incluídos 102 pacientes com suspeita de asma, encaminhados para o Serviço de Pneumologia do HC-UFMG (Hospital das Clínicas Universidade de Minas Gerais), sendo 70 confirmados como asmáticos. A assistência médica anterior foi considerada adequada em apenas 18,6\% dos pacientes. A análise dos dados demonstrou que o manejo de pacientes asmáticos pela maioria dos médicos não-especialistas do sistema público de saúde está em desacordo com as diretrizes, sendo necessários programas de educação médica continuada, disponibilização de medicamentos específicos para o tratamento da asma, treinamentos adequados e divulgação dos conhecimentos disponibilizados pelas diretrizes de asma. ${ }^{19}$

Em 2008, um programa educacional de curta duração melhorou o conhecimento sobre asma, diminuiu mitos e melhorou a habilidade do manejo da asma, proporcionou ao paciente motivação e participação ativa no tratamento da sua 
doença. Foi evidenciado também a redução dos sintomas de dispnéia relacionada ao exercício através do entendimento das diferenças entre o efeito anti-inflamatório e broncodilatador das medicações. ${ }^{20}$

Em 2012, Rodrigues descreveu um estudo em Porto Alegre prospectivo envolvendo pacientes com idade igual ou maior que 14 anos, com asma não controlada, recrutados a partir do ambulatório de um hospital universitário. O estudo foi conduzido antes e depois de uma intervenção educacional para asma. Após uma visita ambulatorial de rotina, os participantes respondiam a questionário estruturado para avaliar o grau de controle da asma, os escores de qualidade de vida (questionário de Juniper) e a técnica inalatória. Os participantes também foram submetidos a testes de função pulmonar. Após, eles participaram de um programa educativo em asma que consistia de uma sessão inicial individualizada de 45 minutos e de entrevistas telefônicas de 30 minutos em 2, 4 e 8 semanas. Esse estudo mostrou que a intervenção educativa resultou no aumento da proporção de pacientes que usava espaçador de $36 \%$ para $68 \%$ e, consequentemente, obtendo maior controle da asma (44,4\% passaram para asma parcialmente controlada e 9,5\% para asma controlada). A proporção de pacientes com técnica inalatória adequada melhorou significativamente de $15,4 \%$ para $46,2 \%(\mathrm{p}=0,021)$ para aqueles em uso do aerossol dosimetrado e de $21,3 \%$ para $76.6 \%(\mathrm{p}<0,001)$ para aqueles em uso de dispositivo em pó. Este programa educativo para pacientes asmáticos ambulatoriais resultou em melhora do grau de controle da doença. Também houve significante

\section{REFERÊNCIAS}

1 Sociedade Brasileira de Pneumologia e Tisiologia. Diretrizes da Sociedade Brasileira de Pneumologia e Tisiologia para o manejo da asma - 2012. J Bras Pneumol. 2012;38 Supl 1:S1-S46.

2 Global Initiative for Asthma. Global strategy for asthma management and prevention. Cape Town (ZA): GINA; 2012 [acesso 2015 jan 28]. 110 p. Disponível em: http://www.ginasthma.org/local/ uploads/files/GINA_Report_March13_1.pdf

3 Tarantino, AB. Doenças Pulmonares. 6. ed. Rio de Janeiro: Guanabara Koogan; 2008. 937 p.

4 Luna MF, Almeida PC, Silva MG. Prevalência de asma em adolescentes na cidade de Fortaleza, CE. J Bras Pneumol. 2009;35(11):1060-67.

5 Behrman RE, Kliegman R, Jenson HB. Nelson: tratado de pediatria. 18. ed. Rio de Janeiro: Elsevier; 2009.

6 Souza GR, Conde MB. Pneumologia e tisiologia: uma abordagem prática. São Paulo: Atheneu; 2009. 635 p.

7 Gibson PG, Powell H, Coughlan J, Wilson AJ, Abramson M, Haywood P, et al. Educación para el autocuidado y examen médico regular para adultos con asma. La Biblioteca Cochrane Plus. 2008;( 2):1-81.

8 Amaral LM, Palma PV, Leite IC. Evolução das políticas públicas e programas de controle da asma no Brasil sob perspectiva dos consensos. J Bras Pneumol. 2012;38(4):518-25. melhora no VEF1 e nos escores de qualidade de vida. A técnica inalatória incorreta na avaliação inicial foi preditora de resposta favorável à intervenção educativa. A implicação clinica do presente estudo é que uma intervenção educativa ambulatorial presencial, seguida de acompanhamento por telefone, melhora significativamente o controle da doença em asmáticos não controlados, principalmente, em decorrência do melhor aprendizado da técnica inalatória. Assim, esse estudo concluiu que um programa educativo individualizado ambulatorial teve efeito positivo sobre o grau de controle da asma com melhora da função pulmonar e melhora nos escores de qualidade de vida. ${ }^{9}$

\section{CONCLUSÃO}

A conscientização e a educação são fatores fundamentais para um adequado controle da doença, possibilitando melhor convívio com esta e tornando o indivíduo capaz de prevenir e detectar as principais complicações.

Para manter a asma sob controle é fundamental que os fatores desencadeantes sejam identificados e que as medidas de profilaxia sejam realizadas.

\section{AGRADECIMENTOS}

Agradeço, em especial, ao Professor Álvaro Madeiro, da Universidade Federal do Ceará, pela revisão do texto.

9 Rodrigues CD, Dalcin PT. O efeito de um programa educativo ambulatorial sobre a asma não controlada. [Dissertação]. Porto Alegre: Universidade Federal do Rio Grande do Sul; 2012. 103 p.

10 Global Initiative for Asthma. Global strategy for asthma management and prevention. Cape Town (ZA): GINA; 2011. 106p.

11 Gillisen A. Patient's adherence in asthma. J Physiol Pharmacol. 2007;58 Suppl 5 Pt 1:205-22.

12 Boulet LP, Vervloet D, Magar Y, Foster JM. Adherence: the goal to control asthma. Clin Chest Med. 2012;33(3):405-17.

13 Burgess S, Sly P, Devadason S. Adherence with preventive medication in childhood asthma. Pulm Med. 2011;2011:1-6.

14 Santana LF, Bastos RC, Martinez-Silveira MS, Souza, LS. Intervenções educativas em asma na infância. Uma revisão analítica da literatura. J Bras Pneumol. 2005;31(5):445-58.

15 Chatkin JM, Cavalet-Blanco D, Scaglia NC, Tonietto RG, Wagner $\mathrm{MB}$, Fritscher CC. Compliance with maintenance treatment of asthma (ADERE study). J Bras Pneumol. 2006;32(4):277-83.

16 Araujo AC, Ferraz E, Borges MC, Filho JT, Vianna EO. Investigation of factors associated with difficult-to-control asthma. J Bras Pneumol. 2007;33(5):495-501.

17 Costa MR, Oliveira MA, Santoro IL, Juliano Y, Pinto JR, Fernandes AL. Educational camp for children with asthma. J Bras 
Pneumol. 2008; 34(4):191-5.

18 Angelini L, Robles-Ribeiro PG, Carvalho-Pinto RM, Ribeiro M, Cukier A, Stelmach R. Two-year evaluation of an educational program for adult outpatients with asthma. J Bras Pneumol. 2009;35(7):618-27.
19 Silveira CD, Araujo FB, Pereira LF, Corrêa RA. Evaluation of the treatment provided to patients with asthma by the Brazilian Unified Heath Care System. J Bras Pneumol. 2009;35(7):628-34.

20 Costa MR, Oliveira MA, Santoro IL, Juliano Y, Pinto JR, Fernandes AL. Acampamento educacional para crianças asmáticas. J Bras Pneumol. 2008;34(4):191-5.

\section{Como citar:}

Barreto AE, Silva FR. Impacto das tecnologias educativas no controle da asma: uma revisão sistemática da literatura. Rev Med UFC. 2015 juldez;55(2):33-38. 